\title{
Perinephric Collection: Hematoma or Duodenal Perforation?
}

\author{
Elsie Ellimah Mensah Zahoor Fazili Lamia Nayeb Howard Marsh
}

Medway Maritime Hospital, Gillingham, Kent, UK

\section{Key Words}

Duodenal perforation

\begin{abstract}
A 74-year-old man presented with a history of collapse and abdominal pain. Initial imaging showed a left sided preirenal collection. The initial working diagnosis was perirenal hematoma secondary to renal trauma. Subsequent patient deterioration led to further imaging which led to the diagnosis of a duodenal perforation with tracking of duodenal contents into the left perirenal space. This important differential should be a consideration in cases of perinephric collections of unknown etiology.
\end{abstract}

Copyright $\odot 2013$ S. Karger AG, Basel

\section{Case Report}

We present the case of a 74-year-old gentleman referred to physicians with collapse and poorly controlled diabetes preceded by a 3-week history of malaise, poor appetite and abdominal pain. He was admitted and treated for possible urosepsis. During his inpatient stay, he developed progressive right sided abdominal pain and abdominal distension. A CT scan (fig. 1) of his abdomen was performed and this showed a perinephric collection reported as a perinephric hematoma. The patient denied any history of abdominal trauma. There was a vague history of collapse but he was quite certain that this did not result in any obvious injuries. There was no associated hematuria and he remained hemodynamically stable. He was referred to the urology team and was managed conservatively as a renal trauma with strict bed rest and antibiotics.

He underwent a follow-up CT scan (fig. 2) 2 days later and this showed the hematoma was not increasing in size. Upon review of the images in a specialist uro-radiology meeting, multiple lacerations of the right kidney were suspected in view of multiple foci of linear hypo enhancement within the right kidney. Management remained unchanged.

The patient continued to have upper abdominal pain and became febrile. A repeat CT scan (fig. 3) demonstrated a communication of the right perinephric collection with the duodenum. The differential diagnosis at this stage was 1: Perinephric hematoma secondary to renal trauma fistulating into the duodenum and 2: Duodenal perforation with collection of upper gastrointestinal (GI) contents into the right perinephric space.

A gastrografin contrast study (fig. 4) also demonstrated leakage of oral contrast into the right perinephric space. The case was discussed in the upper GI multidisciplinary meeting and it was felt that the most likely diagnosis was an upper GI perforation.

The patient was referred to an upper GI center for further management. He underwent an upper GI endoscopy which showed a perforated duodenal ulcer. He was clinically very well at this stage and was managed conservatively with total parenteral nutrition in the initial stages. Progress was monitored with CT and gastrografin contrast studies. A feeding jejunostomy was finally sited for enteral feeding as he remained nil by mouth.

\section{KARGER}

Fax +4161306 1234

E-Mail karger@karger.ch

www.karger.com
(C) 2013 S. Karger AG, Basel

1015-9770/13/0073-0204\$38.00/0

Accessible online at:

www.karger.com/cur
Elsie Ellimah Mensah

Medway Maritime Hospital

Gillingham (UK)

E-Mail eellimah@doctors.org.uk 


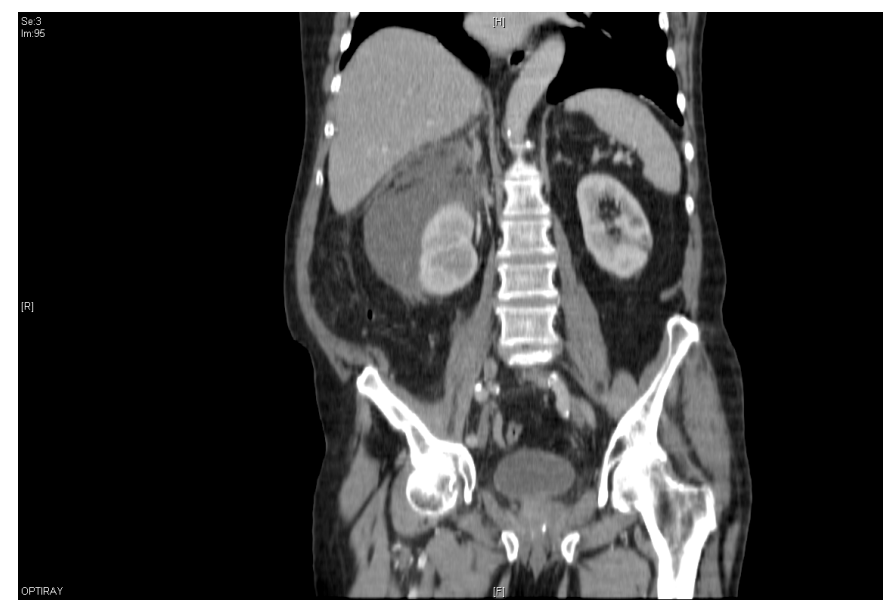

Fig. 1. Right perinephric collection.

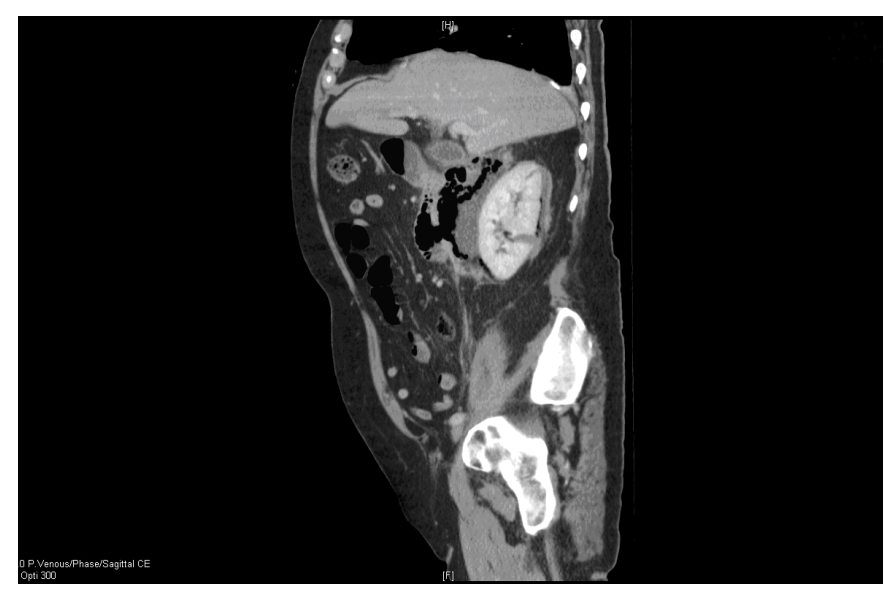

Fig. 3. Sagittal view showing relationship between perinephric hematoma and duodenum.

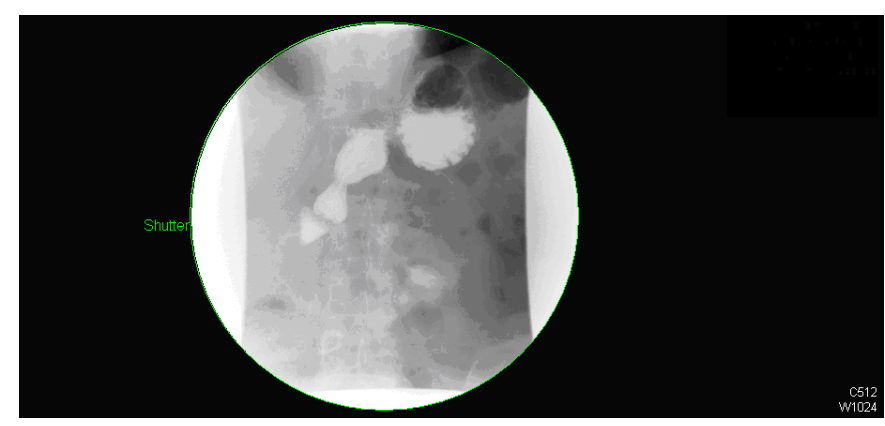

Fig. 5. Follow-up gastrografin study.

Perinephric Collection: Hematoma or Duodenal Perforation?

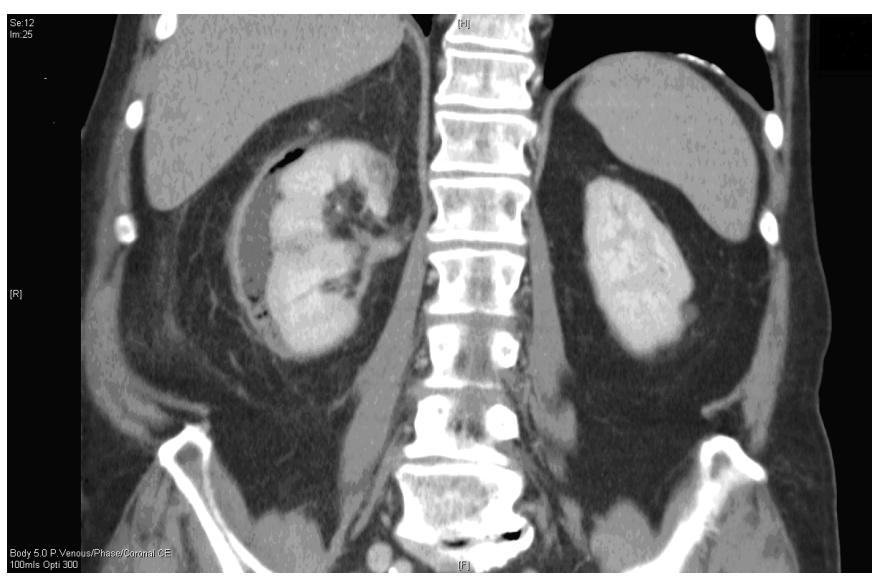

Fig. 2. Right perinephric hematoma - 2 days later.

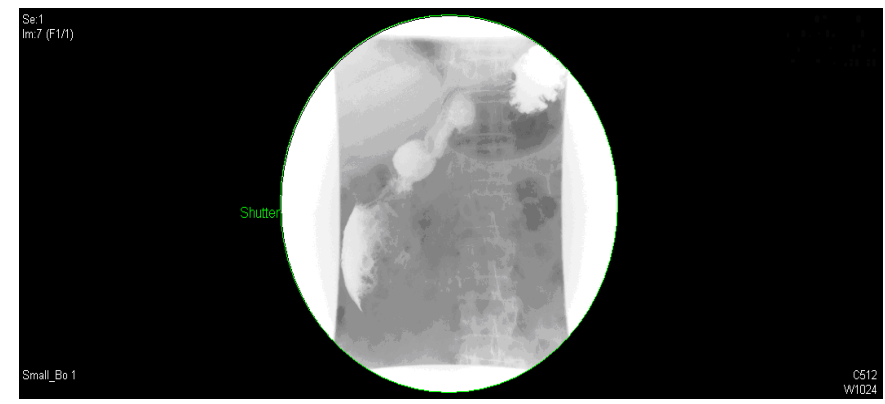

Fig. 4. Gastrografin study showing leakage of ingested contrast into right perirenal space.

Oral nutrition was finally commenced once a gastrografin study showed no further leakage of oral contrast implying that the perforation had finally sealed (fig. 5).

\section{Discussion}

Perirenal hematoma caused by renal trauma is clearly a very important diagnosis to make as delayed diagnosis and inadequate management can result in ongoing bleeding and hemodynamic compromise. Secondly,

Curr Urol 2013;7:204-206 
long-term compression of the kidney by a perirenal collection can have ongoing consequences in the form secondary hypertension secondary to the Page Kidney phenomenon [1]. In the absence of a clear history of trauma however, other causes of collections in the right perirenal space must be considered. There have been a few papers and case reports that demonstrate a relationship between duodenal perforation and changes in the right perirenal space. This ranges from free gas in the right perirenal space to perirenal abscess formation. One study has demonstrated that following posterior duodenal perforation, gas can extend posteriorly into the right perirenal space and this radiological finding can aid diagnosis [2]. In other case reports, this important diagnosis of duodenal perforation has been made at laparotomy following continued clinical deterioration of a patient being treated for a perinephric abscess [3].

This case demonstrates the importance of considering other diagnosis, particularly duodenal perforation when faced with right sided perinephric collections especially in the absence of a corresponding history of trauma. Failure to recognise that the cause of the perinephric collection was a duodenal perforation in this case would have led to clinical deterioration as he would have continued to have oral intake which would have worsened the collection.

\section{References}

1 Oliveira GH, Schirger A: Images in clinical medicine. Page Kidney. N Engl J Med 2003; 348:129.
2 Yagan N, Auh YH, Fisher A: Extension of air into the right perirenal space after duodenal perforation: CT findings. Radiology 2009; 250:740-748.
3 Bapat G, Flynn JT: Posterior duodenal wall perforation presenting as a perinephric abscess. BJU Int 2000;86:1093. 